\title{
Singleton-Merten dysplasia
}

INSERM

\section{Source}

INSERM. (1999). Orphanet: an online rare disease and orphan drug data base. SingletonMerten dysplasia. ORPHA:85191

Sing leton-Merten dysplasia is characterized by dental dysplasia, progressive calcification of the thoracic aorta with stenosis, osteoporosis and expansion of the marrow cavities in hand bones. Additional features included generalized muscle weakness and atrophy, and chronic psoriasiform skin eruptions. It has been reported in four unrelated patients (male and female) and in a family with multiple affected members (male). 